12

\title{
Регистрация спектров инфракрасной фотолюминесценции методом стробируемого интегрирования в режиме активного вычитания фонового сигнала
}

\author{
(С) А.И. Луферов, Д.Д. Фирсов, О.С. Комков
}

Санкт-Петербургский государственный электротехнический университет „ЛЭТИ“, 197376 Санкт-Петербург, Россия

e-mail: andrei.luferau@gmail.com

Поступила в редакцию 27.06.2019 г.

В окончательной редакции 27.06.2019 г.

Принята к публикации 01.10.2019 г.

На основе инфракрасного (ИК) фурье-спектрометра реализован метод, позволяющий проводить измерения ИК фотолюминесценции с высокой скважностью импульсов накачки, что снижает неконтролируемый нагрев полупроводниковых структур возбуждающим лазером. Метод применен для регистрации спектров ИК фотолюминесценции тестовых узкозонных низкоразмерных гетероструктур в диапазоне длин волн 1-5 $\mu \mathrm{m}$. Определено, что при высоких скважностях опорного импульса (больше 20) развиваемый метод стробируемого интегрирования имеет лучшее отношение сигнал-шум в измеряемых спектрах по сравнению с традиционно используемым методом синхронного детектирования.

Ключевые слова: фотолюминесценция, инфракрасный фурье-спектрометр, тепловой фон, стробируемый интегратор, низкоразмерная гетероструктура, синхронный усилитель.

DOI: $10.21883 / O S .2020 .01 .48850 .273-19$

\section{Введение}

Фотолюминесценция (ФЛ) является одним из самых распространенных методов бесконтактного исследования оптических свойств полупроводников [1]. Традиционно для получения спектров инфракрасной (ИК) ФЛ используется методика синхронного детектирования: полезный сигнал регистрируется синхронным (Lock-in) усилителем на частоте модуляции интенсивности источника возбуждения ФЛ. При этом немодулированный тепловой фон, генерируемый нагретыми до комнатной температуры компонентами измерительной схемы, не детектируется.

Методика синхронного детектирования реализуется как на дифракционных спектрометрах, так и на фурьеспектрометрах [2,3]. Последние обладают более высокой эффективностью при измерениях спектров в ИК диапазоне $(\lambda>1.7 \mu \mathrm{m})$ [4]. Основным элементом фурьеспектрометра является интерферометр (в классическом варианте - интерферометр Майкельсона) [5]. Для получения оптического спектра производится фурьепреобразование электрического сигнала, генерируемого фотоприемником при попадании на него проинтерферировавшего светового излучения [6]. При этом в зависимости от характера перемещения подвижного зеркала интерферометра различают два режима работы спектрометра. В режиме пошагового сканирования смена положения зеркала происходит с некоторой временной задержкой в каждом положении, в то время как в режиме быстрого непрерывного сканирования происходит безостановочное перемещение зеркала.
Для реализации методики синхронного детектирования на ИК фурье-спектрометре (FTIR) модуляция возбуждения ФЛ должна проходить на частоте, много большей частоты перемещения подвижного зеркала интерферометра (частоты регистрации экстремумов интерференции опорного внутреннего Не-Ne-лазера). Следовательно, при низких частотах модуляции методика синхронного детектирования может быть реализована только в режиме пошагового сканирования $[7,8]$. В этом режиме полезный сигнал ФЛ выделяется синхронным усилителем на опорной частоте из электрического сигнала фотодетектора в интервале между перемещениями зеркала интерферометра. Интервал выбирается таким образом, чтобы синхронный усилитель мог собрать достаточный объем выборки для последующего усреднения и передачи на вход аналого-цифрового преобразователя (АЦП) фурье-спектрометра.

Принцип работы синхронного усилителя накладывает ограничения на вид опорного сигнала, модулирующего источник возбуждения ФЛ. В идеальном случае этот сигнал должен иметь гармонический вид. В случае использования импульсного сигнала обычный синхронный усилитель распознает лишь первый гармонический член разложения Фурье этого сигнала по опорной частоте [9], что приводит к неизбежной потере уровня полезного сигнала. Действительно, амплитуда первого гармонического члена ряда Фурье импульсного сигнала $A_{1}$ уменьшается с увеличением скважности $S$ согласно формуле [10]

$$
A_{1}=2 A \sin (\pi / S) / \pi,
$$

где $A$ - амплитуда импульсного сигнала. Следовательно, максимальной эффективности синхронного усиления 


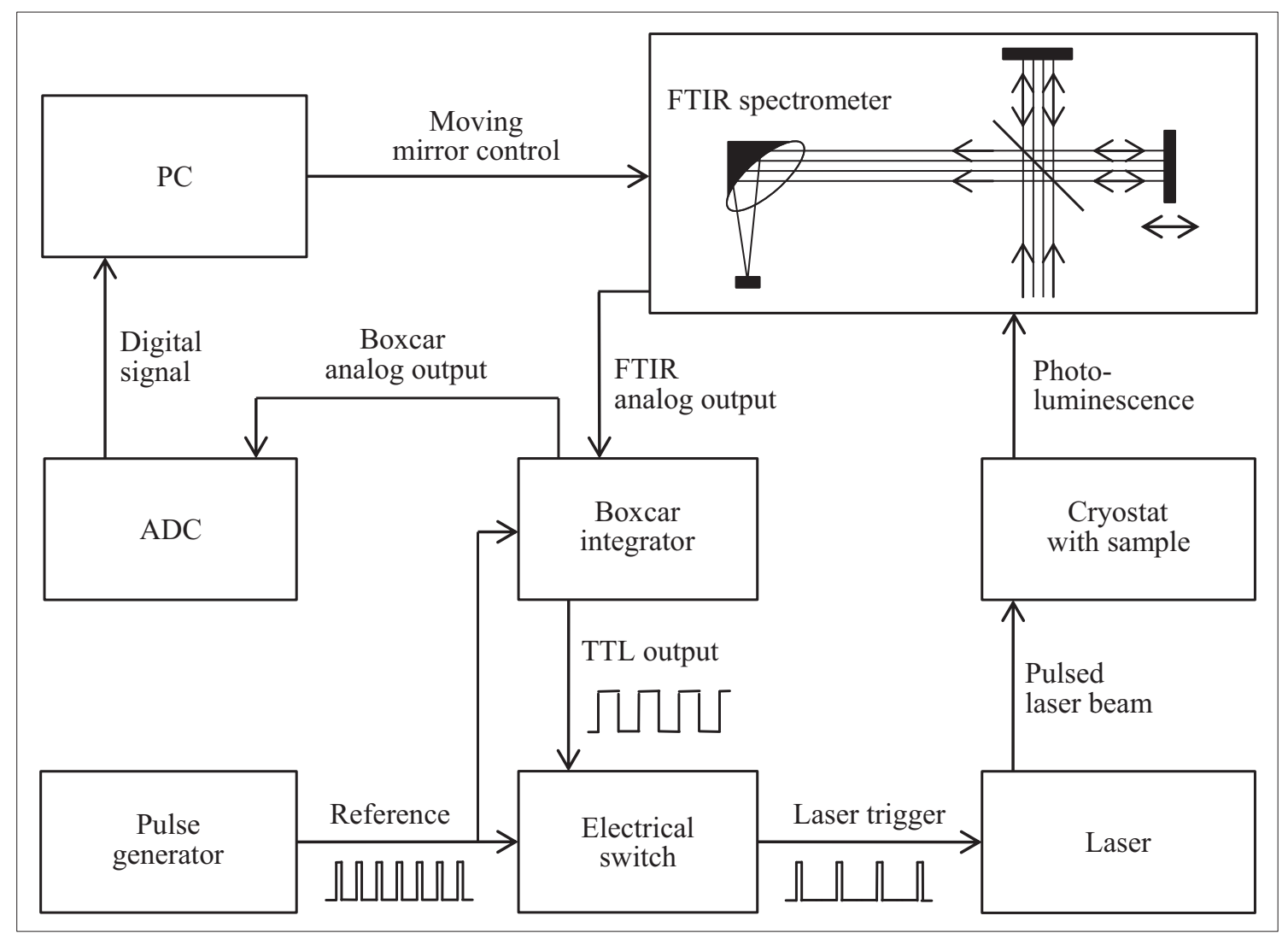

Рис. 1. Блок-схема установки для реализации методики измерений ФЛ со стробируемым интегратором в режиме активного вычитания фонового сигнала.

и соответственно максимального отношения сигнал-шум в получаемых спектрах ИК ФЛ можно добиться, когда форма опорного импульса будет максимально подобна форме гармонической функции.

Режим измерения, при котором лазер накачки модулируется импульсным сигналом малой скважности, приводит к избыточному неконтролируемому нагреву исследуемой структуры [11]. При этом температура ее излучающей области часто остается неизвестной, так как температурный датчик расположен у подложки образца. Точные значения температуры излучающей области структуры необходимы для реализации ряда специальных методик, например для методики определения внутренней квантовой эффективности, описанной в [12]. Для уменьшения избыточного нагрева структуры необходимо уменьшать время засветки структуры лазером накачки путем увеличения скважности опорных импульсов. В таких условиях синхронный усилитель потеряет большую часть сигнала из-за разложения в ряд Фурье, что уменьшает точность результатов.

В настоящей работе описан метод регистрации ИК ФЛ с помощью стробируемого интегратора в режиме активного вычитания фонового сигнала. Данный метод представляет собой альтернативу методу синхронного детектирования в удалении паразитного теплового фона из спектров ИК ФЛ при модуляции лазера накачки импульсами высокой скважности.

\section{Метод стробируемого интегрирования при измерениях ФЛ}

Стробирумый интегратор - прибор, традиционно используемый для регистрации сигналов с временным разрешением $[13,14]$. Благодаря работе с разрешением по времени стробируемый интегратор (в отличие от синхронного усилителя, работающего в частотном разрешении) одинаково хорошо может обрабатывать различные по своей форме и скважности сигналы. В стандартном режиме работы стробируемый интегратор регистрирует весь поступающий электрический сигнал, что в случае измерений ИК ФЛ не позволяет избавиться от паразитного теплового фона. Для этой задачи в работе использован режим активного вычитания фонового сигнала. Блок-схема реализованной установки, работающей в данном режиме, представлена на рис. 1.

Как видно из блок-схемы, сигнал с генератора импульсов является одновременно опорным сигналом для стробируемого интегратора и входным сигналом для элек- 

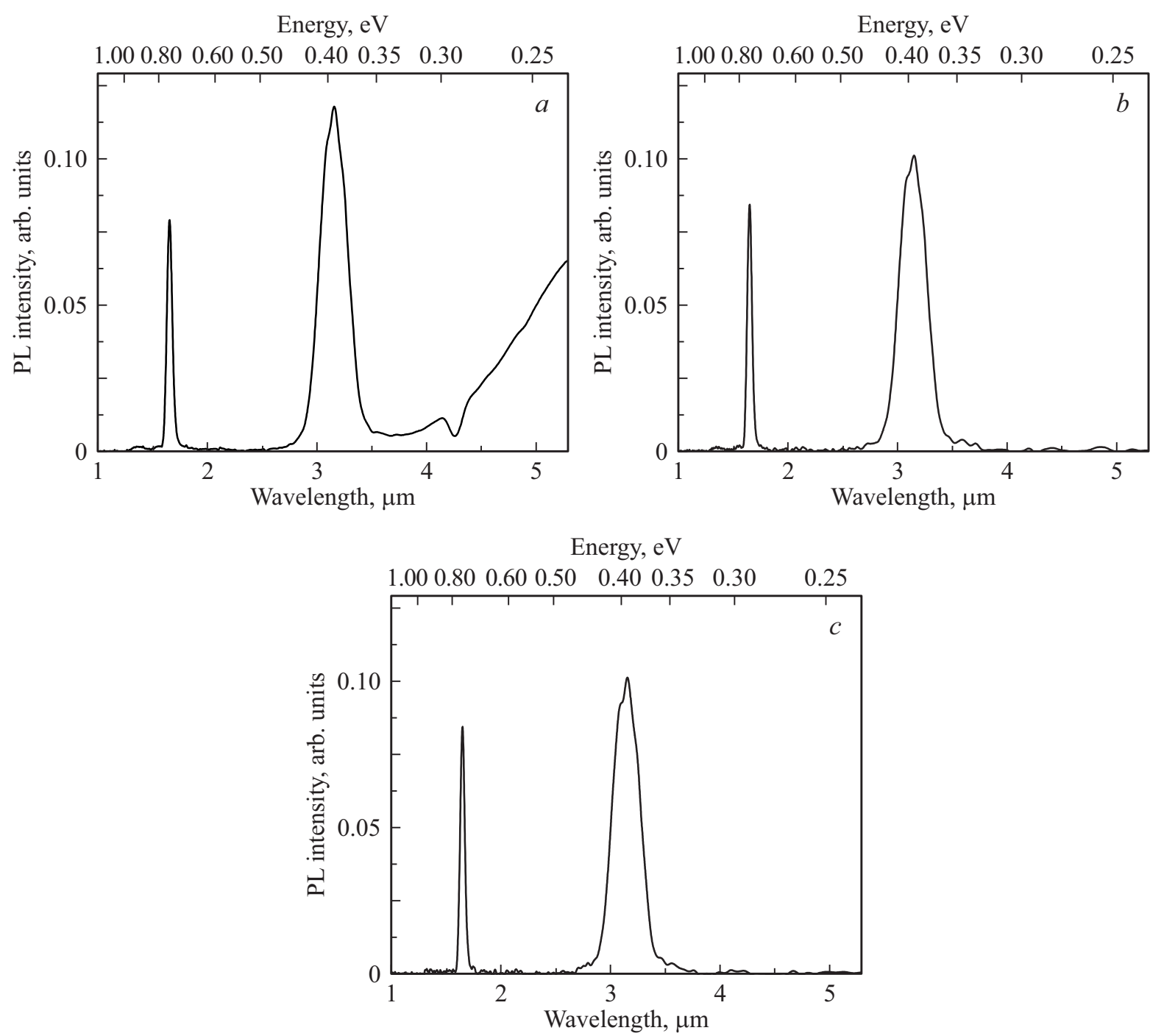

Рис. 2. Спектры ИК ФЛ $(T=8 \mathrm{~K})$ тестовой гетероструктуры, измеренные на ИК фурье-спектрометре: $a-$ в режиме непрерывного сканирования, $b-$ в режиме пошагового сканирования с использованием синхронного усилителя, $c-$ в режиме пошагового сканирования с использованием стробируемого интегратора.

трического ключа. Электрический аналоговый ключ, в свою очередь, управляется транзисторно-транзисторным логическим (ТТЛ) сигналом, который формируется стробируемым интегратором. Логическая единица управляющего сигнала открывает ключ, а логический ноль закрывает. Так как частота ТТЛ сигнала вдвое меньше опорной частоты [15], выходной сигнал электрического ключа позволяет включать лазер „через раз“. Это обеспечивает функционирование режима активного вычитания фонового сигнала стробируемого интегратора: прибор обрабатывает сигнал, детектируемый фотодетектором как при наличии ФЛ, так и тепловой фон в отсутствие ФЛ, затем производит их вычитание. Из полученной разности формируется выборка, которая усредняется. При этом выходное отношение сигнал-шум будет расти в корневой зависимости от объема произведенной выборки [16]. Объем выборки, производимой стробируемым интегратором, пропорционален времени накопле- ния сигнала интегратором и устанавливается вручную на панели прибора.

\section{Результаты}

Используемая в работе экспериментальная установка для регистрации спектров ИК ФЛ реализована на базе ИК фурье-спектрометра VERTEX 80. Модернизация системы регистрации спектрометра [8] позволила выводить усиленный сигнал фотоприемника как на синхронный усилитель SR-830 (Lock-in Amplifier), так и на стробируемый интегратор SR250 (Gated Integrator and BoxcarAverager), а затем вводить в АЦП спектрометра для дальнейшей обработки.

Апробация разработанного метода проводилась на низкоразмерной метаморфной гетероструктуре $\mathrm{InSb} / \mathrm{InAs} / \mathrm{InGaAs} / \mathrm{InAlAs} / \mathrm{GaAs}$, излучающей на длине 


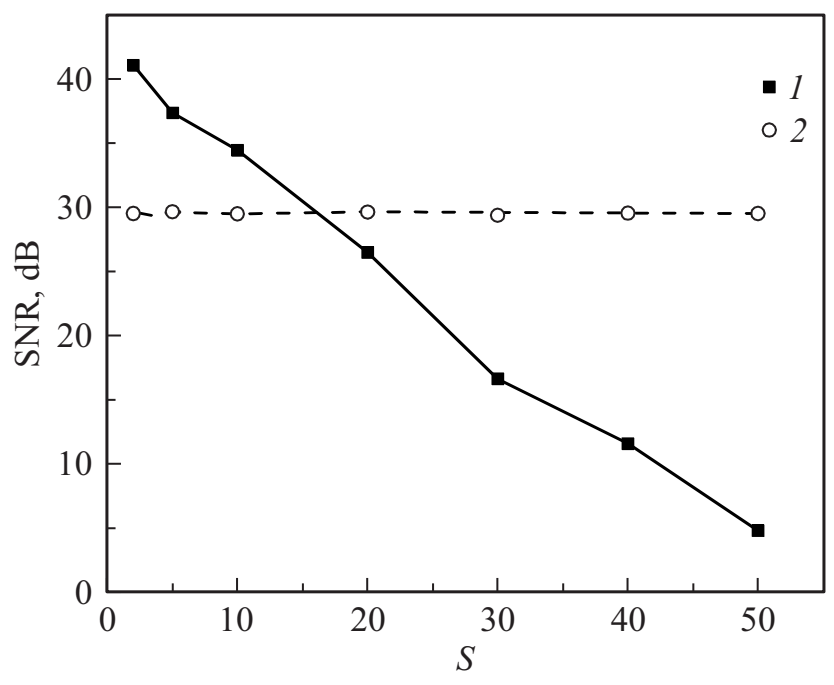

Рис. 3. Экспериментальная зависимость отношения сигналшум ФЛ от скважности сигнала: 1 - синхронный усилитель, 2 - стробируемый интегратор.

волны $\sim 3.3 \mu \mathrm{m}[17,18]$. Для достижения максимальной эффективности в среднем ИК диапазоне измерения производились с использованием $\mathrm{KBr}$-светоделителя и фотоприемника InSb, охлаждаемого жидким азотом. Накачка структуры осуществлялась лазерным диодом на длине волны $809 \mathrm{~nm}$. Для проведения измерений в интервале температур от 8 до $300 \mathrm{~K}$ образец был помещен в гелиевый криостат закрытого цикла Janis CCS- 150 .

На рис. 2, $a$ приведен спектр низкотемпературной ФЛ, полученный в режиме непрерывного сканирования фурье-спектрометра. При этом регистрировался спектр всего излучения, входящего в интерферометр - как ФЛ, так и нежелательного теплового фона (см. длин- новолновую область на рис. 2,a). В нашем случае тестовые структуры обладают достаточно хорошими излучательными характеристиками, и интенсивность ФЛ в диапазоне полезного сигнала $(3.17 \mu \mathrm{m})$ во много раз превышает интенсивность теплового излучения. Однако в противоположной ситуации, когда интенсивность ФЛ исследуемых структур будет мала, получение информации об излучательных переходах в структуре становится невозможным без удаления паразитного теплового фона.

Для получения спектра ФЛ без фонового теплового излучения фурье-спектрометр использовался в режиме пошагового сканирования с применением как метода синхронного детектирования (рис. $2, b$ ), так и метода стробируемого интегрирования (рис. 2,c). Объем выборки, производимой стробируемым интегратором, был выбран таким образом, чтобы время проведения эксперимента с помощью стробируемого интегратора совпадало со временем, затрачиваемым на проведение аналогичных измерений с помощью синхронного детектора.

Из рис. 2 видно, что спектры ИК ФЛ тестовой низкоразмерной гетереструктуры, полученные при помощи как синхронного усилителя, так и стробируемого интегратора, практически совпадают. При этом в обоих спектрах отсутствует паразитное фоновое тепловое излучение $(E<0.34 \mathrm{eV})$, наблюдаемое в спектре, полученном без помощи усилительного оборудования. Это свидетельствует о корректной реализации метода стробируемого интегрирования в режиме активного вычитания фонового сигнала.

Чтобы продемонстрировать предпочтительность использования стробируемого интегратора при модуляции импульсами высокой скважности, были проведены измерения спектров ФЛ с помощью обоих методов в широком диапазоне скважностей сигнала. При этом сравнение эффективности работы усилительного обору-
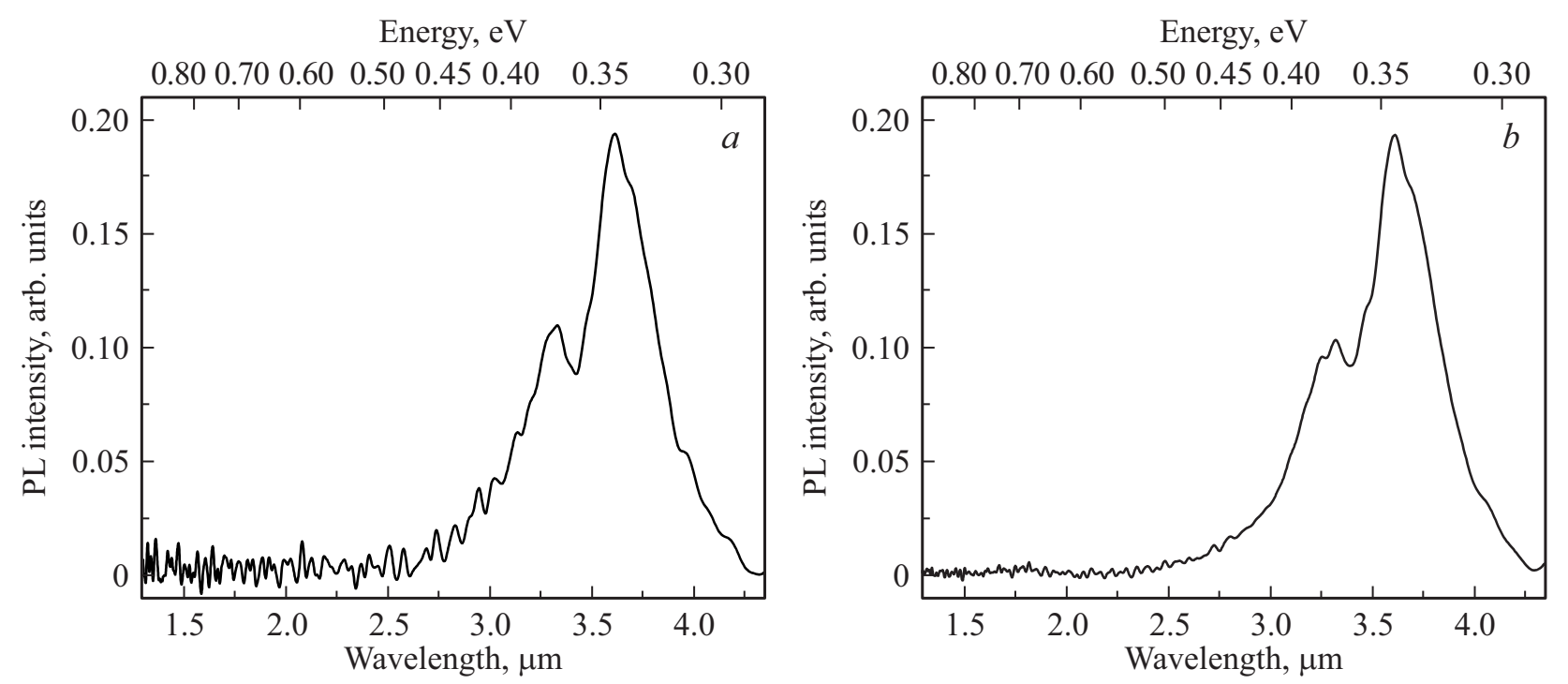

Рис. 4. Спектры ФЛ $(T=300 \mathrm{~K})$, измеренные с помощью усилительного оборудования: $a-$ синхронный усилитель, $b-$ стробируемый интегратор. 
дования проводилось по отношению сигнал-шум (ОСШ) получаемых спектров ФЛ (рис. 3). Как видно из рис. 3, ОСШ спектров, полученных при помощи стробируемого интегратора, не зависит от скважности опорного сигнала, в то время как на синхронном усилителе с увеличением скважности наблюдается значительное снижение ОСШ.

При малых скважностях метод синхронного детектирования демонстрирует лучшие результаты, что обусловливает его повсеместное применение. Однако при высоких скважностях (начиная со значения $S=20$ ) стробируемый интегратор начинает усиливать сигнал ФЛ эффективнее, чем синхронный усилитель. Действительно, при увеличении скважности сигнала от 2 до 50 ОСШ выходного сигнала синхронного усилителя уменьшилось на $\sim 98 \%$. По формуле разложения Фурье амплитуда выходного сигнала при таком увеличении скважности должна уменьшаться на 94\%. Следовательно, уменьшение уровня полезного сигнала на синхронном усилителе с увеличением скважности связано с потерей на разложение Фурье, в то время как уровень полезного сигнала на стробируемом интеграторе стабилен с изменением скважности.

Для визуализации преимуществ использования стробируемого интегратора были произведены измерения спектров ФЛ тестовой структуры при комнатной температуре при скважности $S=50$ (рис. 4). Ввиду малости сигнала ФЛ при $300 \mathrm{~K}$ в полученных спектрах можно визуально определить различающиеся в несколько раз амплитуды шумов.

Для подтверждения уменьшения неконтролируемого нагрева структуры при увеличении скважности импульсов лазера накачки дополнительно были измерены температурные зависимости ФЛ тестовой гетероструктуры при скважности $S=50$. При этом оценивалось изменение отношения интегральной интенсивности ФЛ основного $(3.17 \mu \mathrm{m})$ и неосновного $(1.65 \mu \mathrm{m})$ пиков (рис. 2). Основной пик относительно стабилен по амплитуде [19], и его положение с изменением $T$ меняется слабо [20], в то время как интенсивность неосновного пика с уменьшением температуры сильно растет. Сопоставление экспериментальных зависимостей отношения интегральных интенсивностей пиков от скважности с экспериментальными зависимостями этого же отношения, измеренными при различных температурах, подтвердило уменьшение неконтролируемого нагрева структуры.

\section{Заключение}

Метод стробируемого интегрирования с активным вычитанием фонового сигнала является альтернативой методу синхронного усиления для эффективного подавления паразитного фонового излучения. При малых скважностях синхронный усилитель превосходит стробируемый интегратор как по эффективности, так и по удобству использования, что определяет его широкое применение. Однако увеличение скважности опорного сигнала с 2 до 50 при проведении измерений ФЛ с помощью синхронного усилителя приводит к уменьшению ОСШ на выходе в 70 раз, что делает предпочтительным использование метода стробируемого интегрирования с активным вычитанием фонового сигнала при детектировании ФЛ с большой скважностью.

\section{Благодарности}

Авторы выражают благодарность группе молекулярно-пучковой эпитаксии ФТИ им. А.Ф. Иоффе РАН под руководством С.В. Иванова за выращивание тестовых структур.

\section{Финансирование работы}

Работа выполнена при поддержке гранта Российского научного фонда (проект № 18-79-10161).

\section{Конфликт интересов}

Авторы заявляют, что у них нет конфликта интересов

\section{Список литературы}

[1] Cardona M. Fundamentals of Semiconductors. Berlin: Springer, 2010. 778 p. doi 10.1007/978-3-642-00710-1

[2] Imasaka T., Yoshitake A., Ishibashi N. // Anal. Chem. 1984. V. 56. N 7. P. 1077-1079. doi 10.1021/ac00271a007

[3] Mandon J., Guelachvili G., Picqué N. // Nat. Phot. 2009. V. 3. N 2. P. $99-102$.

[4] Hosea T.J.C., Merrick M., Murdin B.N. // Phys. Stat. Sol. (a). 2005. V. 202. N 7. P. 1233-1243. doi 10.1002/pssa.200460908

[5] Матвеев А.Н. Оптика. М.: Высш. шк., 1985. 351 с.

[6] Ефимова А.И., Зайцев В.Б., Болдырев Н.Ю., Кашкаров П.К. Оптика: инфракрасная фурье-спектрометрия. М.: Юрайт, 2018. 143 с.

[7] Chernov M.Yu., Solov'ev V.A., Komkov O.S., Firsov D.D., Meltser B.Ya., Yagovkina M.A., Baidakova M.V., Kop'ev P.S., Ivanov S.V. // Appl. Phys. Express. 2017. V. 10. N 12. P. 121201. doi 10.7567/APEX.10.121201

[8] Фирсов Д.Д., Комков О.С. // Письма в ЖТФ. 2013. T. 39. № 23. C. 87-94; Firsov D.D., Komkov O.S. // Tech. Phys. Lett. 2013. V. 39. N 12. P. 1071-1073. doi 10.1134/S1063785013120079

[9] Stanford Research Systems. Model SR830 DSP Lock-in Amplifier: Operation and Service Manual. Sunnyvale, CA: Author, 2011. 178 p.

[10] Жук В.В., Натансон Г.И. Тригонометрические ряды Фурье и элементы теории аппроксимации. Л.: Изд-во Ленингр. ун-та, 1983. 188 с.

[11] Mironova M.S., Komkov O.S., Firsov D.D., Glinskii G.F. // J. Phys.: Conf. Ser. 2014. V. 541. N 1. P. 012085. doi 10.1088/1742-6596/541/1/012085

[12] Yoo Y.S., Roh T.M., Na J.H., Son S.J., Cho Y.H. // Appl. Phys. Lett. 2013. V. 102. N 21. P. 211107. doi 10.1063/1.4807485

[13] Novo J.B.M., Pessine F.B.T. // Appl. Spec. 1993. V. 47. N 12. P. 2044-2051. 
[14] Vij D.R. Luminescence of Solids. N.Y:: Springer US, 1998. 427 p. doi $10.1007 / 978-1-4615-5361-8$

[15] Stanford Research Systems. Model SR250 Gated Integrator and Boxcar Averager Module: Operation and Service Manual. Sunnyvale, CA: Author, 1993. 157 p.

[16] Omenetto N. Analytical Laser Spectroscopy. N.Y.: Springer US, 1985. 290 p. doi 10.1007/978-1-4613-2441-6

[17] Chernov M.Yu., Komkov O.S., Firsov D.D., Meltser B.Ya., Semenov A.N., Terent'ev Ya.V., Brunkov P.N., Sitnikova A.A., Kop'ev P.S., Ivanov S.V., Solov'ev V.A. // J. Cryst. Growth. 2017. V. 477. P. 97-99. doi 10.1016/j.jcrysgro.2017.02.017

[18] Ivanov S.V., Chernov M.Yu., Solov'ev V.A., Brunkov P.N., Firsov D.D., Komkov O.S. // Progr. in Cryst. Growth and Characterization of Materials. 2019. V. 65. P. 20-35. doi 10.1016/j.pcrysgrow.2018.12.001

[19] Komkov O.S., Firsov D.D., Chernov M.Yu., Solov'ev V.A., Sitnikova A.A., Kop'ev P.S., Ivanov S.V. // J. Phys. D: Appl. Phys. 2018. V. 51. N 5. P. 055106. doi 10.1088/13616463/aaa25d

[20] Firsov D.D., Komkov O.S., Solov'ev V.A., Kop'ev P.S., Ivanov S.V. // J. Phys. D: Appl. Phys. 2016. V. 49. N 28. P. 285108. doi 10.1088/0022-3727/49/28/285108 